\title{
miR-1284 modulates multidrug resistance of gastric cancer cells by targeting EIF4A1
}

\author{
WENLONG CAO $^{1}$, WEIYUAN WEI ${ }^{1}$, ZEXU ZHAN $^{1}$, \\ $\mathrm{YUBO}^{\mathrm{XIE}}{ }^{2}$ and QIANG XIAO ${ }^{1}$ \\ Departments of ${ }^{1}$ Surgery and ${ }^{2}$ Anaesthesiology, The First Affiliated Hospital of \\ Guangxi Medical University, Nanning, Guangxi 530021, P.R. China
}

Received November 6, 2015; Accepted December 18, 2015

DOI: $10.3892 /$ or.2016.4643

\begin{abstract}
Routine chemotherapy as an important treatment mode often can not be effective because of multidrug resistance (MDR). MicroRNA (miRNA) modulates the expression of a great number of genes, including MDR. In this study, the expression of miR-1284 was reduced in gastric cancer (GC) tissue specimens with metastasis and in vincristine-resistant (VCR) GC SGC7901 cells (SGC-7901/VCR) compared to that in the controls. Recombinant lentiviral vectors with miR-1284 led to the overexpression of miR-1284 mRNA and reversed the chemoresistance of SGC7901/VCR cells, promoted cell cycle arrested at the G0/G1 phase, accelerated drug-induced apoptosis, and decreased migration and invasiveness of SGC-7901/VCR. In addition, the overexpression of miR-1284 sensitized tumors to chemotherapy in vivo. Our data provide combined evidence that miR-1284 can heighten the expression of MYC and reduce the expression of JUN, MMP12, and EIF4A1 that was the direct target. In conclusion, miR-1284 can function as a new regulator to reduce GC MDR cells by targeting EIF4A1.
\end{abstract}

\section{Introduction}

Gastric cancer (GC) is one of the most universal malignancies, however the advances in recent decades have improved long-

Correspondence to: Professor Qiang Xiao, Department of Surgery, The First Affiliated Hospital of Guangxi Medical University, 6 Shuang-yong Road, Nanning, Guangxi 530021, P.R. China

E-mail: xiaoqiang20050@aliyun.com

Professor Yubo Xie, Department of Anaesthesiology, The First Affiliated Hospital of Guangxi Medical University, 6 Shuang-yong Road, Nanning, Guangxi 530021, P.R. China

E-mail: xieyubo715001@aliyun.com

Abbreviations: $\mathrm{MDR}$, multidrug resistance; GC, gastric cancer

Key words: miR-1284, EIF4A1, gastric carcinoma, drug resistance, murine model term survival only slightly (1). Surgery and chemotherapy are the main protocols for GC therapy; however, in many cases, the efficacy of even the leading chemotherapy treatment is poor, regardless of whether it is natural or acquired (2). The term 'multidrug resistance (MDR)' is used to define this phenomenon and could provide an insight into the reasons for the low 5-year survival rate of GC patients $(3,4)$. Pervasively, the molecular mechanisms underlying MDR are complex, covering intricate courses, including drug conveyance and drug-induced apoptosis (5). Until now, the mechanisms responsible for MDR in GC have not been adequately determined.

MicroRNAs (miRNAs) are a type of non-coding RNAs that are approximately 19-24 nucleotides long and that downregulate the expression of genes by targeting the 3'-untranslated regions (3'-UTRs) of particular mRNAs that are situated in the genomic areas that are exclusively related to carcinoma $(6,7)$, miRNAs are consequently considered to be connected with chemotherapy failure, and emerging evidence shows that MDR may be regulated by altering miRNA (8-10). For example, miR-23b-3p sensitizes GC cells to chemical agents by regulating ATG12 and HMGB2 (11); overexpression of $\mathrm{miR}-181 \mathrm{~b}$ and $\mathrm{miR}-497$ was able to increase the sensitivity of cells to chemotherapy by targeting $\mathrm{Bcl} 2$, known as an anti-apoptotic gene $(12,13)$. Patnaik et al (14) proved that the expression of miR-1284 is much lower in lung adenocarcinoma than in their controls. Recent research also suggested that compared with primary GC, miR-1284 is downregulated in GC with lymph node metastases (15); however, there is still lack of evidence as to the precise role of miR-1284 in MDR in GC.

To define the effects of miR-1284 in GC MDR, we determined miR-1284 expression in GC tissue specimens with metastasis and the vincristine-resistant (VCR) GC cell line SGC7901 (SGC7901/VCR). In addition, we established the SGC-7901/VCR cells with a stable overexpression of miR-1284 and probed alterations in $\mathrm{IC}_{50}$, cell cycles, apoptosis, and migration and invasiveness. We also investigated the influence of miR-1284 overexpression on tumor growth in vivo. To ensure that the underlying mechanism was identified, we surveyed the impact of miR-1284 on the expression of genes associated with MDR; apoptosis; and migration, including EIF4A1. 


\section{Materials and methods}

Ethics statement. The study was approved by the Ethics Board of The First Affiliated Hospital of Guangxi Medical University and complied with the Declaration of Helsinki. All the patients gave their written informed consent. The animal procedures were conducted following the provisions of the Ethics Committee of The First Affiliated Hospital of Guangxi Medical University in the research. All efforts were made to minimize suffering.

Human tissue samples and cell lines. Gastric cancer tissues and adjacent non-tumor tissues (located $5 \mathrm{~cm}$ away from the tumor) were collected during surgery in The First Affiliated Hospital of Guangxi Medical University (during in 2013-2015) and preserved in liquid nitrogen. Vincristineresistant SGC7901 (SGC7901/VCR) cells were obtained from the Cell Bank of the Chinese Academy of Sciences (Shanghai, China). SGC7901/VCR cells were cultivated in RPMI-1640 (GE Healthcare Life Sciences, South Logan, UT, USA), with $50 \mathrm{mg} / \mathrm{ml}$ penicillin, $100 \mathrm{mg} / \mathrm{ml}$ streptomycin, $10 \%$ fetal bovine serum, and $0.8 \mu \mathrm{g} / \mathrm{ml}$ vincristine to maintain drug resistance. Cells were cultured at $37.8^{\circ} \mathrm{C}$ with $5.0 \%$ $\mathrm{CO}_{2}$.

Antibodies. Primary antibodies to EIF4A1 (1:1,000), JUN $(1: 1,000)$, MMP12 (1:1,000), MYC $(1: 1,000)$, and GAPDH $(1: 1,000)$ were provided by Abcam (Cambridge, UK). Secondary antibodies $(1: 10,000)$ were provided by LI-COR Biosciences (Lincoln, NE, USA).

Quantitative reverse transcription real-time polymerase chain reaction. RNA was extracted from SGC7901/VCR cells using TRIzol (Invitrogen Corporation, Carlsbad, CA, USA). cDNA was reverse transcribed from 1,000 ng RNA using the PrimeScript $^{\mathrm{TM}} \mathrm{RT}$ reagent kit (Takara Bio, Inc., Tokyo, Japan). The mRNA or miRNA expression levels were calculated by reference GAPDH (mRNA) or U6 (miRNA). All primer sequences, including those for miR-1284, U6, EIF4A1, JUN, MMP12, MYC, and GAPDH, are listed in Table I. Quantitative reverse transcription real-time polymerase chain reaction (qRT-PCR) methods were developed on a SYBR ${ }^{\circledR}$ Premix Ex Taq ${ }^{\mathrm{TM}}$ II (Tli RNaseH Plus) and a ROX Plus reagent kit (Takara Bio, Inc.) according to the manufacturer's instructions. The mRNA and miRNA expressions were analyzed using the $2^{-\Delta \Delta \mathrm{CT}}$ method.

Transfection of cell lines. The miR-1284 overexpression vector LV-miR-1284-GFP and the null vector LV-GFP were provided by GeneChem (Shanghai, China). After seeded into 6-well plates for $36 \mathrm{~h}$, cells were infected with the lentiviral vector at $100 \mathrm{PFU} / \mathrm{cell}[$ multiplicity of infection $(\mathrm{MOI})=100$ ] without penicillin or streptomycin. To acquire stably transfected SGC7901/VCR cells, the cells were cultured in $600 \mathrm{mg} / \mathrm{ml}$ G418 (Invitrogen Corporation) for 14-21 days. Three groups of cells were identified as follows: gastric cancer SGC7901/VCR cells in the LV-miR-1284-GFP group were transfected with the recombinant lentivirus vector LV-miR-1284-GFP; the cells in the LV-GFP group were transfected with the negative control lentiviral vector LV-GFP, and the cells in the control group
Table I. The sequences of primers for quantitative reversetranscriptase real-time polymerase chain reaction.

\begin{tabular}{ll}
\hline Gene & \multicolumn{1}{c}{ Primer sequences } \\
\hline miR-1284 & F: 5'-CGTCTATACAGACCCTGGCTTTTC-3' \\
& R: 5'-CTCAACTGGTGTCGTGGA-3' \\
U6 & F: 5'-TTATGGGTCCTAGCCTGAC-3' \\
& R: 5'-CACTATTGCGGGTCTGC-3' \\
EAF4A1 & F: 5'-ATCCCAGAGGCTCTCCTCAC-3' \\
& R: 5'-CTACCATTTTCTCTCCCCTGCTT-3' \\
JUN & F: 5'-ACCAAGAACTGCATGGACCTAACA-3' \\
& R: 5'-GCTCAGCCTCGCTCTCACAA-3' \\
MMP12 & F: 5'-ACGTGGCATTCAGTCCCTGT-3' \\
& R:5'-AACACTGGTCTTTGGTCTCTCAGAA-3' \\
MYC & F: 5'-GCAGCTGCTTAGACGCTGGA-3' \\
& R: 5'-CGCAGTAGAAATACGGCTGCAC-3' \\
GAPDH & F: 5'-GCACCGTCAAGGCTGAGAAC-3' \\
& F: 5'-TGGTGAAGACGCCAGTGGA-3'
\end{tabular}

F, forward; R, reverse.

were without any treatment. qRT-PCR was used to detect the miR-1284 in the transfected cells.

Cytotoxicity assay. Cells were implanted into 96-well plates at a density of $2.0 \times 10^{3}$ cells/well. After $24 \mathrm{~h}$, vincristine was divided into six concentrations $(0,0.2,0.4,0.8,1.6$, and $3.2 \mathrm{mg} / \mathrm{ml}$ ) and each was added to a common medium. After incubation for $48 \mathrm{~h}, 10 \mu \mathrm{l}$ Cell Counting Kit-8 reagent (Dojindo, Tokyo, Japan) were added and development was sustained for $1 \mathrm{~h}$ at $37.8^{\circ} \mathrm{C}$ with $5.0 \% \mathrm{CO}_{2}$. The absorbance was detected at $450 \mathrm{~nm}$, and $\mathrm{IC}_{50}$ was calculated using the relative survival curve. Each survey was processed in quadruplicate.

Cell cycle analysis. Cells were washed twice with PBS and fastened with $70 \%$ ethanol for $12 \mathrm{~h}$ at $4^{\circ} \mathrm{C}$. The cells were hatched in a solution that contained $200 \mathrm{ng} / \mathrm{ml}$ RNase and $0.05 \mathrm{mg} / \mathrm{ml}$ propidium iodide that was incubated at ambient temperature for $0.5 \mathrm{~h}$. The results were tested by flow cytometry (BD Biosciences, Mountain View, CA, USA).

Apoptosis assay. Apoptosis was tested using the Apoptosis Detection kit (BD Biosciences) and following the manufacturer's instructions. The cells were hatched in a solution containing $5 \mu \mathrm{l} / \mathrm{ml}$ Annexin V-PE and $5 \mu \mathrm{l} / \mathrm{ml}$ 7-amino-actinomycin $\mathrm{D}$ at $4^{\circ} \mathrm{C}$ in the dark. The results were tested by flow cytometry (BD Biosciences).

Wound-healing assay. To inhibit cell proliferation, cells were cultivated in 6-well plates with mitomycin C. A straight line as a wound was created using a $200-\mu l$ sterile pipette tip and washed twice lightly with PBS to remove any floating cells. The wound conditions were noted at 0,48 , and $96 \mathrm{~h}$ by microscope and the relative motility was calculated using the 
following formula: Relative motility $=($ initial distance - a time point distance)/initial distance $\mathrm{x} 100 \%$.

Cell invasion assay. RPMI-1640 medium (75 $\mu \mathrm{l})$ without serum containing $1 \mu \mathrm{g} / \mathrm{ml}$ Matrigel (BD Biosciences) was used in the above chamber $(6.5 \mathrm{~mm}$; Corning, New York, NY, USA). RPMI-1640 medium $(200 \mu \mathrm{l})$ without fetal calf serum containing $5.0 \times 10^{4}$ cells were placed into the upper chamber, while $700 \mu \mathrm{l}$ RPMI-1640 with $5.0 \%$ fetal calf serum was placed in the lower chamber. After culturing for $24 \mathrm{~h}$, the cells in the lower chamber were stained with Giemsa, and the number of visible cells was counted in six random views at a x200 magnification.

Luciferase reporter assays. Wild-type (WT) EIF4A1 3'UTR, mutated (MUT) EIF4A1 3'UTR, negative (NC) EIF4A1 3'UTR luciferase reporter vector and miR-1284 mimic, and NC miRNA plasmid were purchased from GeneChem. The WT 3'UTR and MUT 3'UTR luciferase reporter vector were co-transfected with miR-1284 mimic while the NC 3'UTR + miR-1284 mimic was used as the control. The $293 \mathrm{~T}$ cells were seeded into 24 -well plates $\left(2.0 \times 10^{4}\right.$ cells/well). After $24 \mathrm{~h}$, the cells were separated into groups and transfected with $0.1 \mu \mathrm{g}$ WT 3'UTR + $0.3 \mu \mathrm{g}$ miR-1284 mimic, $0.1 \mu \mathrm{g}$ MUT 3'UTR $+0.3 \mu \mathrm{g}$ miR-1284 mimic, and $0.1 \mu \mathrm{g} \mathrm{NC}$ 3'UTR $+0.3 \mu \mathrm{g}$ miR-1284 mimic. NC miRNA and the same 3'UTR were co-transfected as a reference in each group. After $48 \mathrm{~h}$, firefly luciferase luminescence activity was assayed using the Dual Luciferase Reporter Assay system (Promega, Madison, WI, USA). Renilla luciferase luminescence was then detected after adding Stop \& Glo ${ }^{\circledR}$ reagent (Promega) to each well. Luminescence was calculated as follows: Relative luciferase activity = firefly luciferase luminescence/Renilla luceriferase luminescence. Each evaluation was processed in triplicate.

Western blot analysis. Proteins were extracted using cell lysate extraction (Solarbio, Beijing, China), separated by sodium dodecyl sulfate-polyacrylamide gel electrophoresis, and then shifted onto nitrocellulose membranes. The membranes were immersed with the antibody $(1: 1,000)$ overnight at $4^{\circ} \mathrm{C}$ and then washed with Tris-buffered saline and Tween-20. The membrane was immersed with a dilution of infrared-labeled secondary antibody $(1: 10,000)$ for $1 \mathrm{~h}$ and Odyssey (LI-COR Biosciences) was used to analyze the optical density. In addition, the expression of GAPDH $(1: 1,000)$ was used as a reference.

Effect of miR-1284 on GC cells in vivo. BALBC/c nude mice, aged 5-6 weeks, were provided by Guangxi Animal Center (Nanning, China), retained in specific-pathogen free surroundings, and cared for by following the instructions of the Ethics Committee of Guangxi Medical University. Tumors were implanted by injecting $4.0 \times 10^{7}$ SGC-7901/VCR cells resuspended in $100 \mu \mathrm{l}$ phosphate-buffered saline (PBS; Beyotime Institute of Biotechnology, Shanghai, China) into the armpit region of the mice. After 4 days, the tumors increased to $\sim 5.0 \mathrm{~mm}$ in diameter and the animals were separated into the following three groups (6 mice/group): LV-miR-1284-GFP, LV-GFP, and control. The tumors were injected with LV-miR-
1284-GFP or LV-GFP at a titer of $5.0 \times 10^{6} \mathrm{TU}$ in $150 \mu \mathrm{l}$ PBS; a similar volume of PBS was injected into the control group. VCR (200 ng/kg) were injected into the peritoneum. Subsequent to the first surgery, the mice were given the same treatment every 2 days. Tumor size was calculated every 4 days using a Vernier caliper and the length diameter (a) and the width diameter (b) were surveyed (tumor volume = $a \times b^{2} / 2$ ). The relative tumor volume (RTV) was estimated by $\mathrm{RTV}=\mathrm{V}_{\mathrm{t}} / \mathrm{V}_{0}\left(\mathrm{~V}_{0}\right.$, the tumor volume at time of intraperitoneal injection; $V_{t}$, the tumor volume at the next measurement). After 20 days of feeding, the animals were sacrificed and the tumors were evaluated. The tumors were immersed in $4 \%$ formaldehyde, water was removed using an ethanol slope, and tumors were inlayed in paraffin. Tumor segments were dewaxed, rehydrated, and stained with hematoxylin and eosin. Sections were observed under a microscope on middle power (x200).

Statistical analyses. SPSS 13.0 (SPSS Inc., Chicago, IL, USA) was used to analyze the data. Data are shown as the mean $\pm \mathrm{SE}$ and deemed to have statistical significance when $\mathrm{P}<0.05$ using Student's t-test, one-way analysis of variance, or $\chi^{2}$ test.

\section{Results}

miR-1284 decreases in gastric cancer tissue specimens and drug resistant $G C$ cells. To determine whether miR-1284 is associated with the evolution of GC with distant metastasis and MDR in GC cells, we detected miR-1284 expression in GC tissue specimens from patients with distant metastasis and compared it to those with adjacent non-tumor tissues. The data from 16 gastric cancer patients showed significant downregulation of miR-1284 in GC tissue specimens $(\mathrm{P}<0.05)$ (Fig. 1A). In addition, the data showed that miR-1284 expression in SGC7901/VCR were different from that of SGC7901 with significant downregulation of miR-1284 in SGC7901/VCR $(\mathrm{P}<0.05)$ (Fig. 1B). Our data indicated that miR-1284 may be related to GC with distant metastasis and MDR in GC cells.

miR-1284 recombinant lentiviral vectors leads to overexpression of $m i R-1284$. To test the hypothesis that miR-1284 may overcome MDR in GC cells, we first established GC cells that stably overexpressed miR-1284. LV-miR-1284-GFP and LV-GFP were transfected into SGC7901/VCR cells, respectively. The expression of miR-1284 in the LV-miR-1284-GFP group was substantially upregulated $(\mathrm{P}<0.05)$ but there was no difference in miR-1284 levels between the LV-GFP and control groups $(\mathrm{P}>0.05)$ (Fig. 1C). The data demonstrated that LV-miR-1284-GFP could upregulate miR-1284 expression in SGC7901/VCR.

miR-1284 overcomes MDR in GC cells. We next examined the impact of miR-1284 overexpression on MDR in GC cells. The sensitivity of SGC7901/VCR cells and IC $_{50}$ values of each group were measured by CCK-8 assay. The LV-miR-1284-GFP group demonstrated greatly enhanced sensitivity to vincristine, as indicated by decreased $\mathrm{IC}_{50}$ values $(\mathrm{P}<0.05)(\mathrm{Fig} .1 \mathrm{D})$. These results proved that miR-1284 overexpression is associated with the GC MDR phenotype. 
A
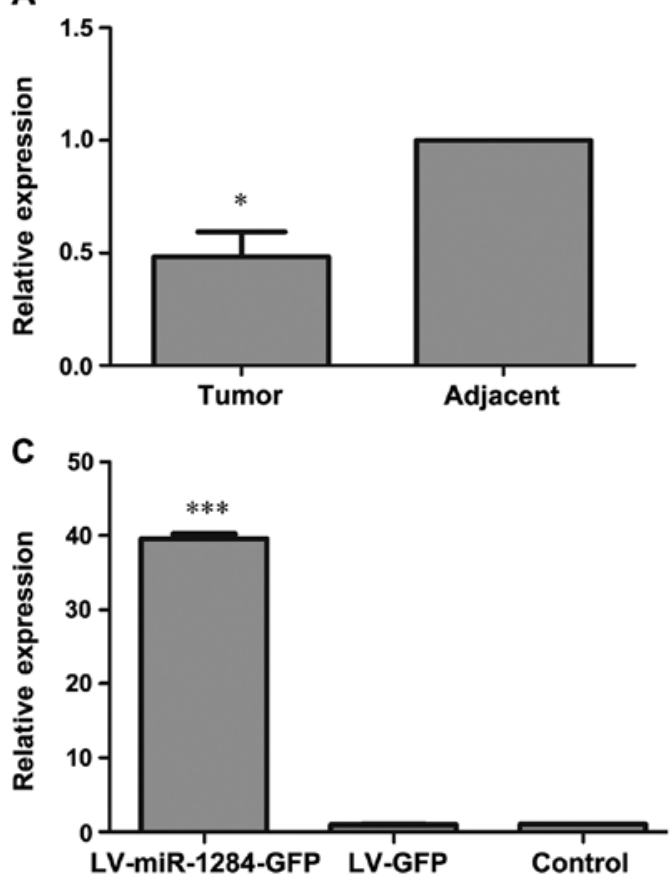

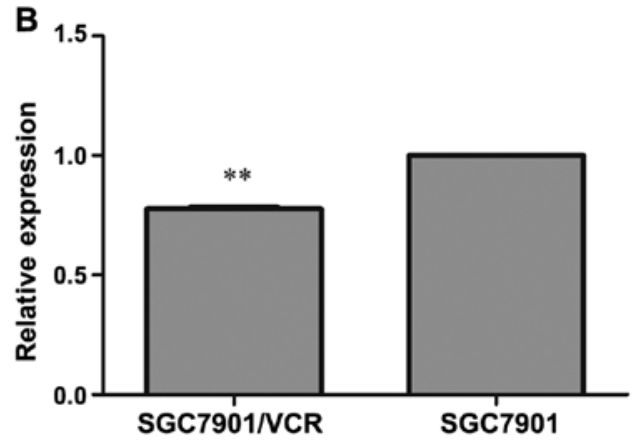

D

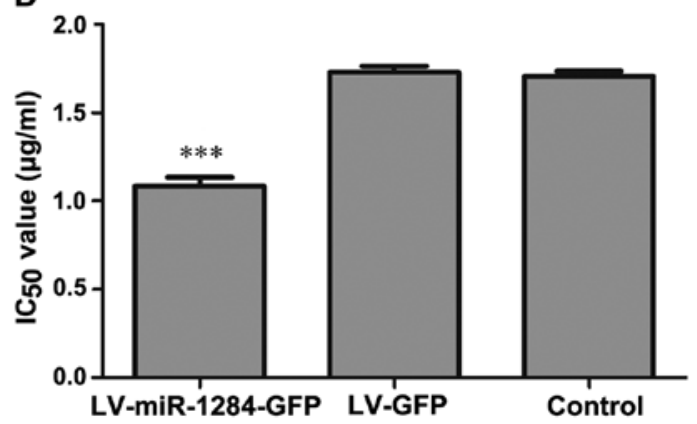

Figure 1. miR-1284 is decreased in gastric cancer (GC) tissues with distant metastasis and multiple drug resistant (MDR) GC cells and overcomes MDR in GC cells. (A) Quantitative reverse transcription real-time polymerase chain reaction (qRT-PCR) of miR-1284 expression in GC tissues from patients with distant metastasis and adjacent non-tumor tissues ( $n=4)$. (B) qRT-PCR of miR-1284 expression in SGC7901/VCR and SGC7901 (n=4). (C) qRT-PCR of the expression of miR-1284 after transfection $(\mathrm{n}=4)$. (D) $\mathrm{IC}_{50}$ values for vincristine $(\mathrm{n}=4)$. ${ }^{*} \mathrm{P}<0.05$ for gastric cancer tissue specimens vs. adjacent non-tumor tissues, ${ }^{* *} \mathrm{P}<0.05$ for the SGC7901/VCR group vs. the SGC7901 group, ${ }^{* * *} \mathrm{P}<0.05$ for the LV-miR-1284-GFP group vs. the LV-GFP and control groups. All values are the mean $\pm \mathrm{SE}$.

miR-1284 prevents cells from entering the $S$ phase. To determine whether miR-1284 overexpression can reverse MDR by effecting the cell cycle, we counted the cells in specific phases. The data showed that cell counts in the G0/G1 phase observably increased, while those in the $\mathrm{S}$ phase decreased in the LV-miR-1284-GFP group $(\mathrm{P}<0.05)$ (Fig. 2A). By miR-1284 preventing GC MDR cells from entering into the $\mathrm{S}$ phase, the GC MDR cells are reduced.

miR-1284 accelerates drug-induced apoptosis. Changes in drug-induced apoptosis can influence the efficacy of chemotherapy drugs; therefore, we looked into the influence of miR-1284 on apoptosis of GC cells induced by chemotherapy. After incubation, VCR cells were detected by flow cytometry. The data show that after miR-1284 overexpression, the apoptosis rate significantly increased $(\mathrm{P}<0.05)$ (Fig. 2B). These results suggested that miR-1284 may increase the apoptosis rate of SGC7901/VCR cells induced by chemotherapy drugs.

miR-1284 decreases the migration of GC cells. To further determine how miR-1284 influences the migration of SGC7901/ VCR cells, we investigated the migratory ability of the GC cells in each group using the wound-healing assay. After $96 \mathrm{~h}$, the cells in the LV-miR-1284-GFP group showed lower migratory ability than those in the LV-GFP and control groups (relative motility rate of cells in the LV-miR-1284-GFP vs. LV-GFP and control groups: $51.02 \pm 2.18 \%$ vs. $82.21 \pm 1.25 \%$ and $82.55 \pm 4.35 \%$, respectively; $\mathrm{P}<0.05$ ) (Fig. $2 \mathrm{C}$ ). The data demonstrated that miR-1284 decreases the migration of GC cells.
miR-1284 decreases the invasion of GC cells. After determining that miR-1284 inhibits the migratory ability of SGC7901/VCR cells, we evaluated whether it may also suppress cell invasion. The Transwell assay demonstrated that fewer cells passed through the membrane of the Matrigel chamber in the LV-miR-1284-GFP group than through the chamber of the LV-GFP and control groups. The number of LV-GFP and control group cells that passed through the membrane was 3.5- and 3.3-fold greater, respectively, than that of the LV-miR-1284-GFP (P<0.05) (Fig. 2D). The data demonstrated that miR-1284 decreases the invasion of GC cells.

EIF4A1 is the direct target gene of miR-1284. The results of TargetScan 6.2 indicated that EIF4A1 is one of the underlying targets of miR-1284 (Fig. 3A). To confirm this, the relative luciferase activity of each group was assayed. The data showed that the relative luciferase activity in the WT 3'UTR + miR-1284 mimic group declined in relation to that in the NC 3'UTR + miR-1284 and MUT 3'UTR + miR-1284 groups $(\mathrm{P}<0.05)$ (Fig. 3B). The results suggested that miR-1284 modulates MDR by targeting EIF4A1.

miR-1284 modulates $M D R$ by reducing the expression of EIF4A1, JUN, and MMP12 and enhancing the expression of $M Y C$. To investigate the mechanism by which miR-1284 reverses MDR in SGC7901/VCR, we used qRT-PCR and western blotting to assess the expression levels of genes that regulate apoptosis, metastasis, and MDR. In the LV-miR-1284-GFP group, the expressions of MYC mRNA 


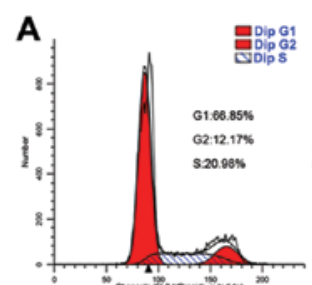

LV-miR-1284-GFP
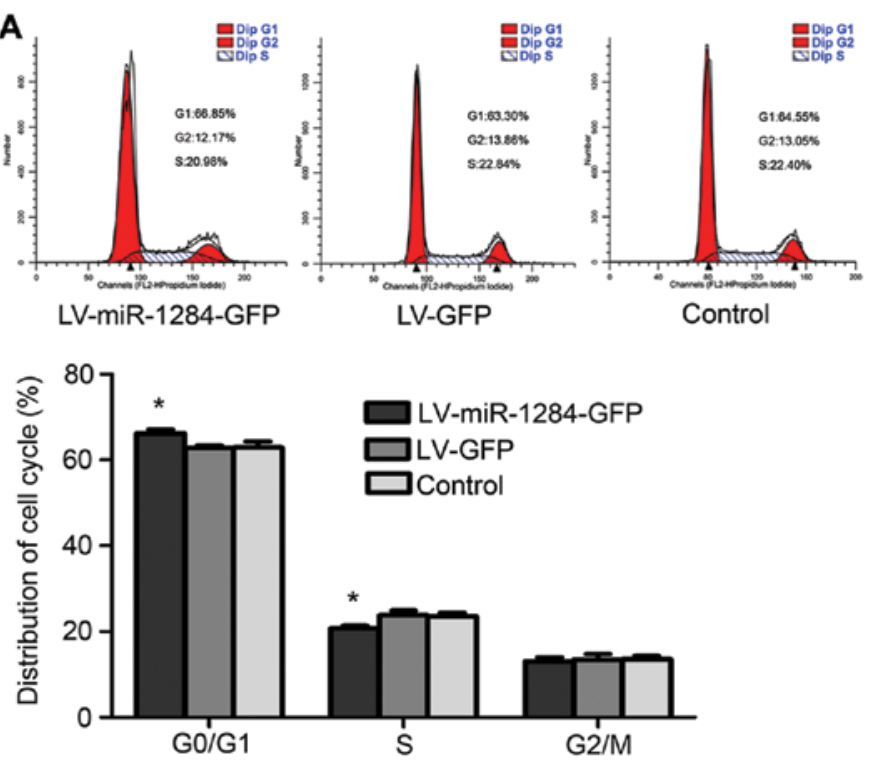

B
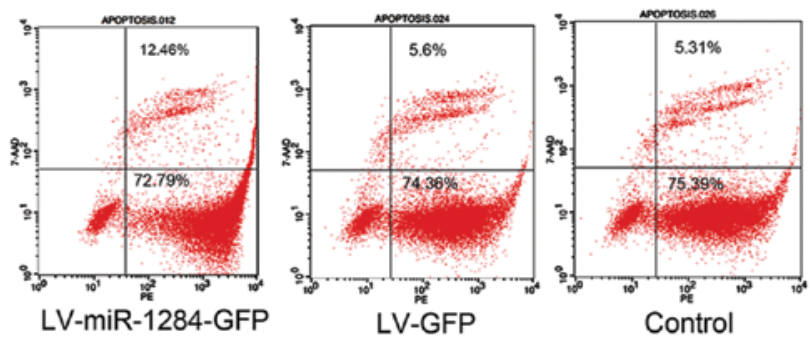

LV-GFP
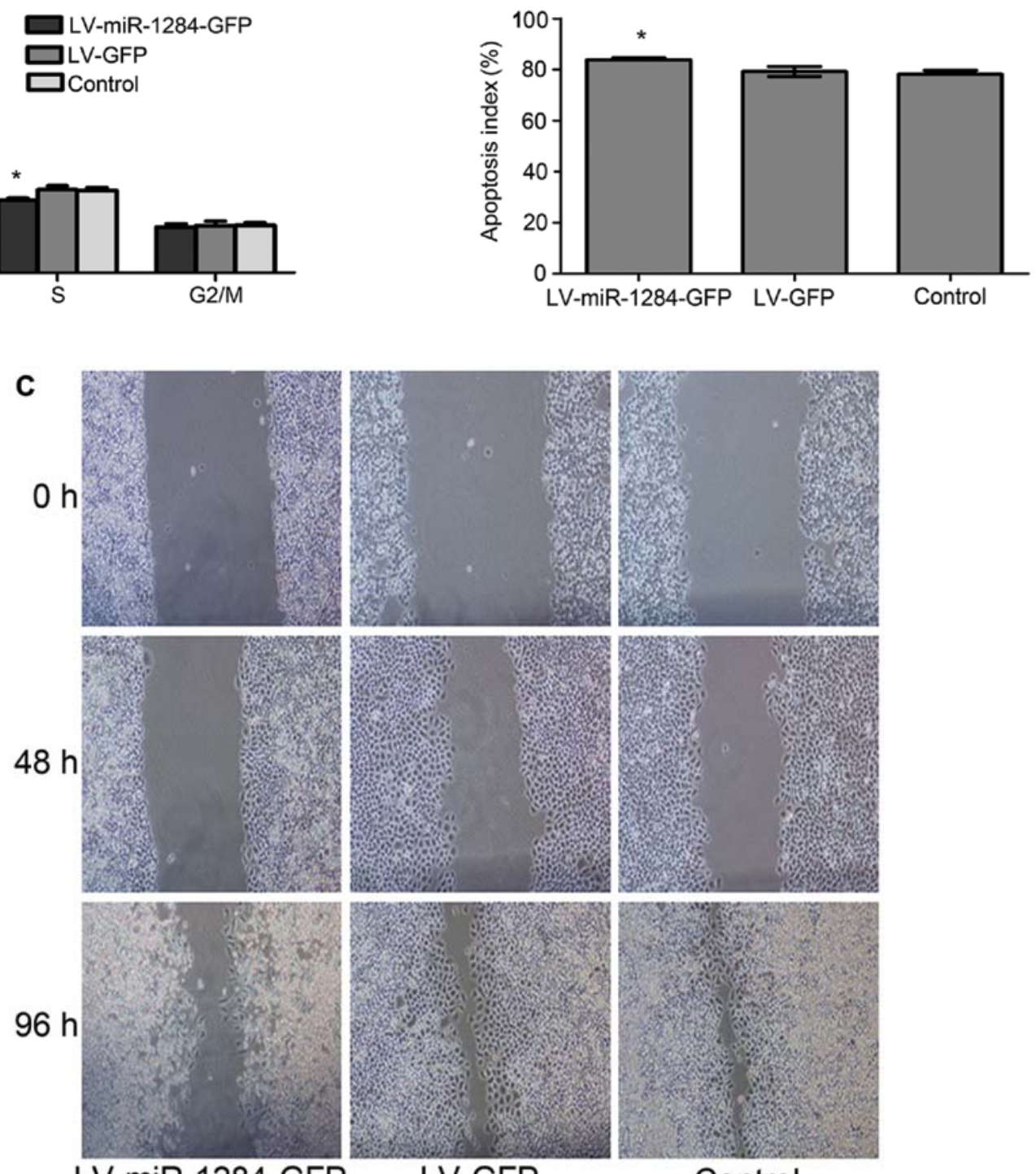

LV-miR-1284-GFP

LV-GFP

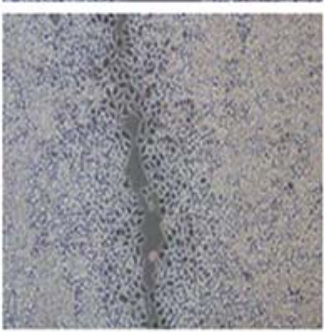

Control

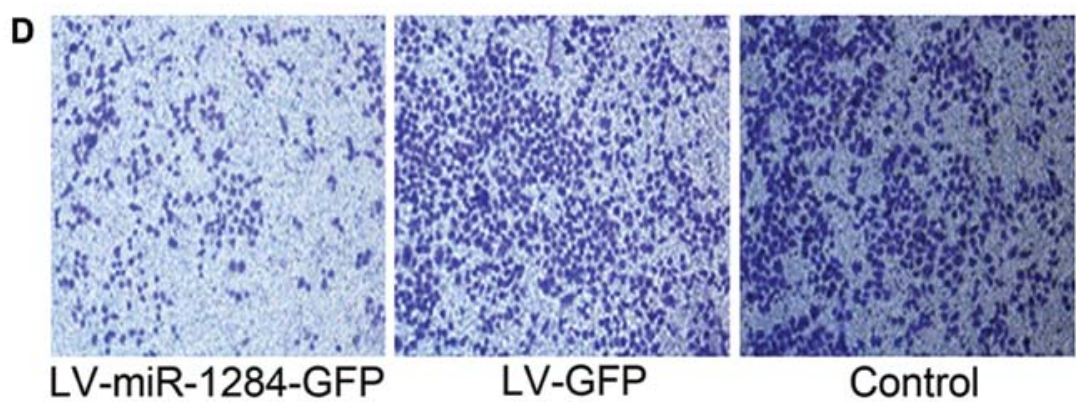

Figure 2. miR-1284 prevents cells from entering into the $\mathrm{S}$ phase, accelerates drug-induced apoptosis, and decreases migration and invasion of gastric cancer (GC) cells. (A) Flow cytometry of the SGC7901/VCR cell cycle after miR-1284 transfection and vincristine infection (n=4). (B) Flow cytometry of the percentages of apoptotic SGC7901/VCR cells after miR-1284 transfection and vincristine infection $(n=4)$. (C) Wound-healing assay $(x 100)$ of the migratory ability of SGC7901/VCR cells after miR-1284 transfection (n=3). (D) Transwell assay (x200) of the invasion of SGC7901/VCR cells after miR-1284 transfection ( $\mathrm{n}=6$ ). ${ }^{*} \mathrm{P}<0.05$ for the LV-miR-1284-GFP group vs. the LV-GFP and control groups. All values are the mean $\pm \mathrm{SE}$. 


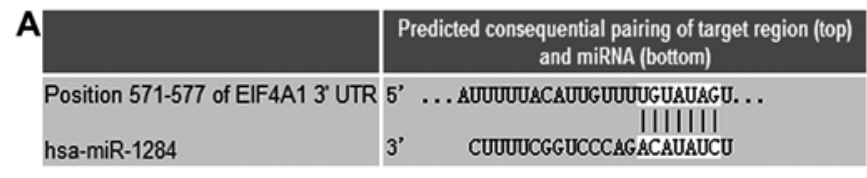

B
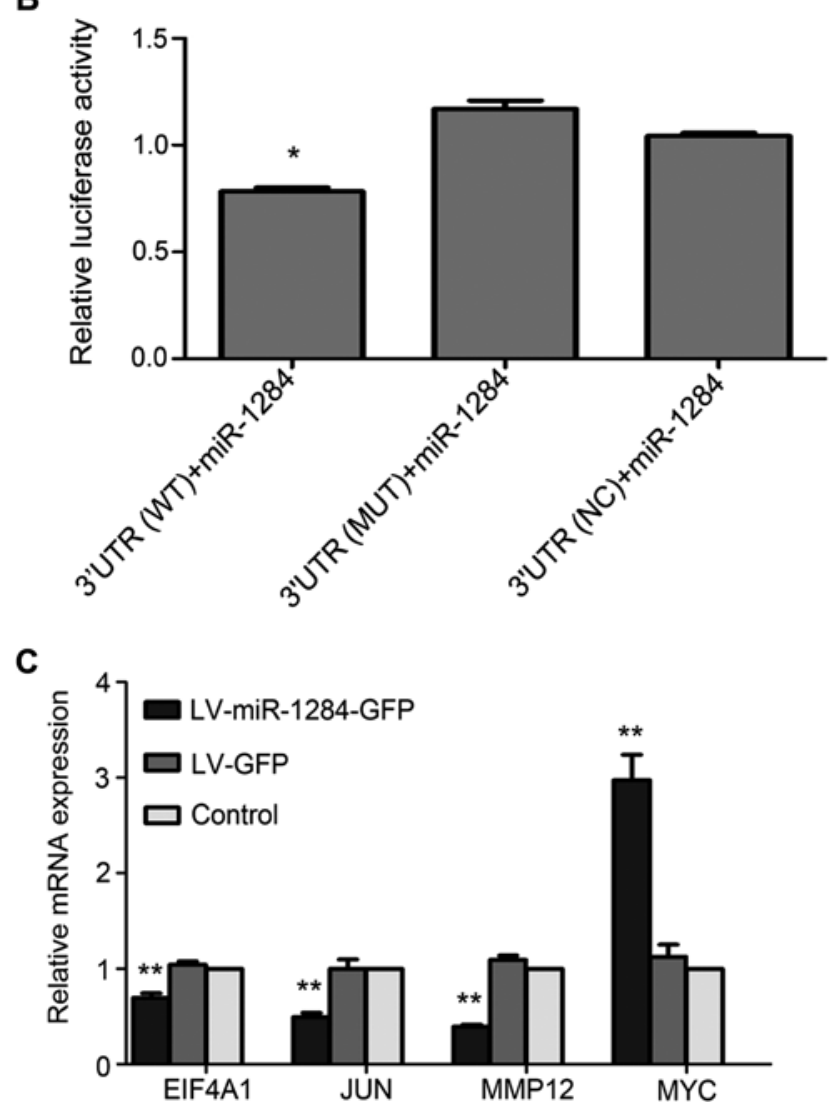

and protein were higher, and the expression of EIF4A1, JUN, and MMP12 lower than those in the LV-GFP and control groups $(\mathrm{P}<0.05)$ (Fig. 3C and D).

miR-1284 reverses $M D R$ in $G C$ in vivo. We also explored the influence of miR-1284 on SGC7901/VCR cells in vivo by subcutaneously transplanting tumors in nude mice. After 4 days, the mice were randomly divided into the LV-miR-1284-GFP group, LV-GFP group, and control group. Each group was injected with LV-miR-1284-GFP, LV-GFP, or PBS, respectively, and given an intratumoral vincristine treatment. After 20 days of treatment, the RTV in the LV-miR-1284-GFP group was significantly smaller than that in either the LV-GFP or control group $(\mathrm{P}<0.05)$ (Fig. 4A). qRT-PCR confirmed that the expression of miR-1284 in the LV-miR-1284-GFP group was greater than that in either the LV-GFP or control group $(\mathrm{P}<0.05)$ (Fig. 4B). The data demonstrated that miR-1284 can reverse MDR in GC in vivo.

\section{Discussion}

GC is the second leading cause of carcinoma death worldwide (16). Due to lack of efficacious methods of diagnosing the early stages of the disease and tumorigenesis, patients who suffer from metastasis lose their best opportunity for
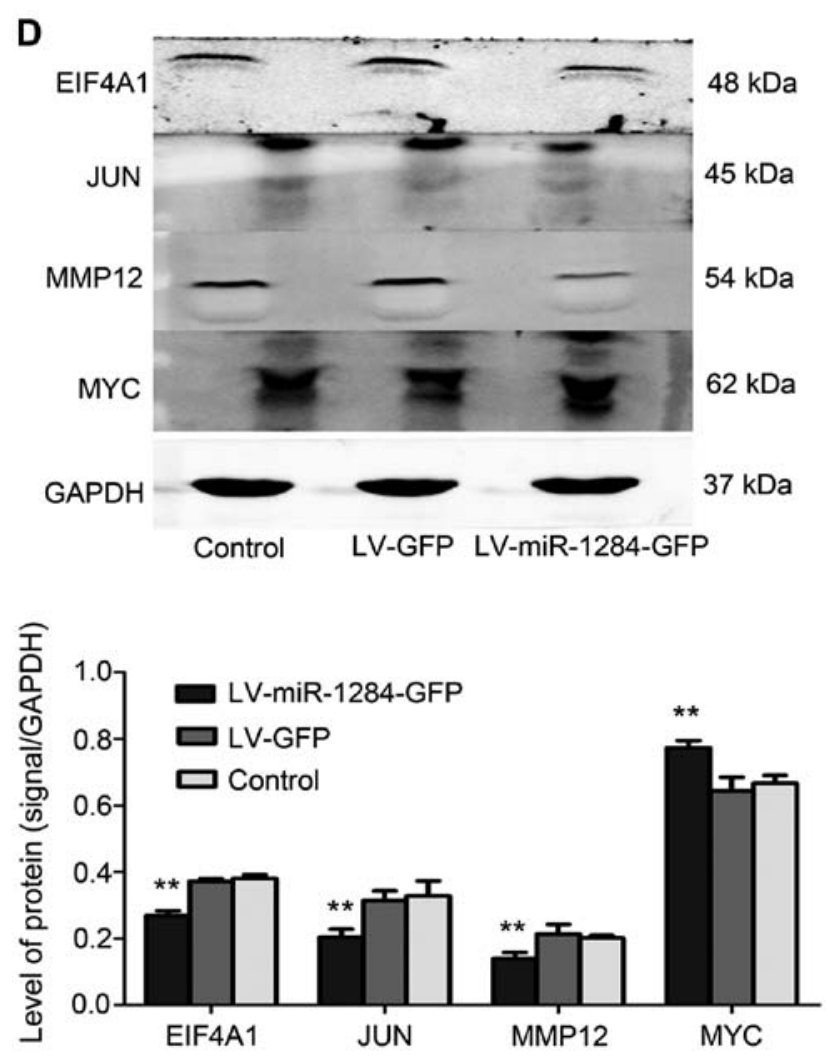

Figure 3. miR-1284 modulates multiple drug resistance (MDR) by targeting EIF4A1 and influences the expression of JUN, MMP12, and MYC. (A) The results of TargetScan forecasted the target gene of miR-1284. (B) The wild-type (WT) 3'UTR and mutant (MUT) 3'UTR luciferase reporter vector were co-transfected with miR-1284 mimic while the negative (NC) 3'UTR + miR-1284 mimic was used as a control. Luciferase activity assay was measured $(n=3)$. (C) Quantitative reverse transcription real-time polymerase chain reaction (qRT-PCR) of EIF4A1, JUN, MMP12, and MYC mRNA ( $n=4)$. (D) Western blotting of EIF4A1, JUN, MMP12, and MYC proteins $(\mathrm{n}=3)$. ${ }^{*} \mathrm{P}<0.05$ for WT 3'UTR + miR-1284 vs. NC 3'UTR + miR-1284 and MUT 3 'UTR + miR-1284, ${ }^{* *} \mathrm{P}<0.05$ for the LV-miR-1284-GFP group vs. LV-GFP and control groups. All values are the mean $\pm \mathrm{SE}$.

surgery, and chemotherapy becomes the preferred treatment; however, routine chemotherapy often fails because of MDR in GC cells (17). Many mechanisms underlying MDR have been extensively explored, including ejecting several drugs into the cells, modifying drug targets, destroying the balance of damaging or repairing DNA, and deactivating drug-induced apoptosis pathways (18-22), however, the crucial factors of this phenomenon remain largely unclear.

Recently, increasing number of studies have revealed that miRNAs modulate the development of MDR in GC (23-25). Chen et al (15) reported that compared with primary GC, miR-1284 is downregulated in GC with lymph node metastases. Even so, the properties and the potential mechanisms by which miR-1284 affects MDR have not been investigated. The current study showed that miR-1284 was reduced in GC specimens with distant metastasis and in SGC7901/ VCR cells compared with that in the controls, which agree with a previous study (15); however, there are no previous studies that compared the connection between miR-1284 and tumorigenesis in GC. In addition, our data showed that modulation of miR-1284 overexpression could overcome MDR in 
A

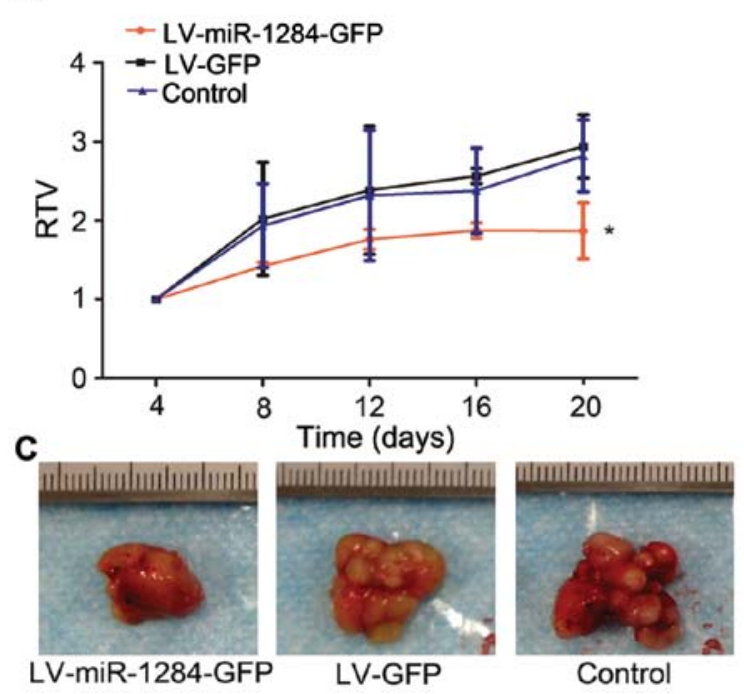

B

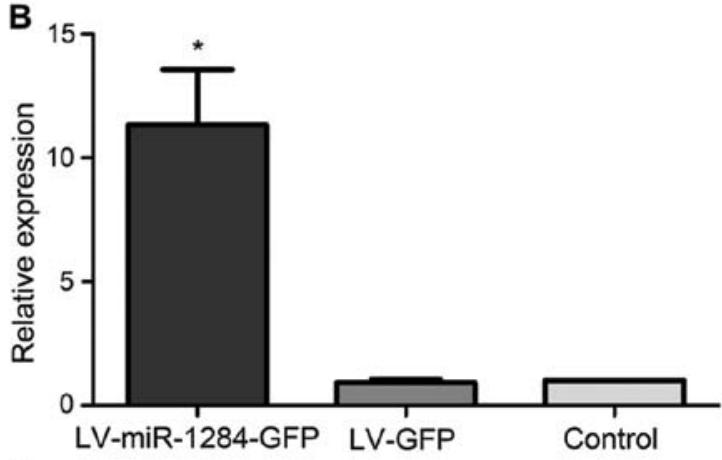

D

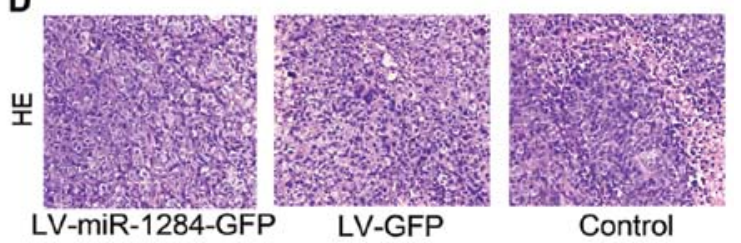

Figure 4. miR-1284 reverses multiple drug resistance (MDR) in gastric cancer (GC) cells in vivo. (A) Relative tumor volume (RTV) of each group (n=6). (B) Quantitative reverse transcription real-time polymerase chain reaction (qRT-PCR) of the miR-1284 expression in each group ( $\mathrm{n}=4$ ). (C) Xenografts of each group. (D) Tumor segments stained with hematoxylin and eosin (x200). Notes: * $<0.05$ for the LV-miR-1284-GFP group vs. the LV-GFP and control groups. All values are the mean \pm SE.

SGC7901/VCR, prevent cells from entering into the S phase, and induce cell apoptosis. miR-1284 overexpression also decreases the migration and invasion of SGC7901/VCR and reverses MDR in vivo, as demonstrated by suspended tumor growth in nude mice. The results confirm that miR-1284 may function as a new regulator to reverse MDR in GC cell line SGC7901/VCR in vitro and in vivo.

Nonetheless, the accurate method by which miR-1284 induces these events is obscure. MDR is reversed by cell death and is associated with EIF4F, a compound that induces ribosomes to gather to mRNA templates, which comprise EIF4E, EIF4G, and EIF4A subunits (26). This study proved that miR-1284 directly both regulates EIF4A1 and suppresses EIF4A1 expression. EIF4A1 is a member of the translation initiation composite EIF4A, which controls protein synthesis, and has been reported to have an indispensable function in tumorigenesis (27). As a DEAD-box helicase, EIF4A1 plays an important role in unwinding integrated RNA components within 5'UTR to gather ribosomes and participate in mRNA translation (28). Increasing number of studies have confirmed that EIF4A1 has multiple functions and is correlated with the genesis and progression of tumor-promoting proteins. Overexpression of EIF4A1 has been proven in many cancers, such as liver cancer, melanoma, and melanocytic nevi $(29,30)$. In addition, recent research has shown that the presence of EIF4A1 can predict an independently adverse outcome in breast cancer and that reducing the expression of EIF4A1 diminishes cell proliferation and prevents cells from entering the $\mathrm{S}$ phase (31). Bordeleau et al (26) demonstrated that the absence of EIF4A1 decreases drug resistance to doxorubicin, which is associated with PI3K/mTOR activation. This is consistent with our findings that miR-1284 reverses MDR in GC by targeting EIF4A1. To our knowledge, this study is the first to demonstrate that EIF4A1 is immediately downregulated by miR-1284 in GC MDR cells.
By exploring the mechanisms of synergistic interaction we demonstrated that miR-1284 overexpression can regulate the response of SGC7901/VCR cells to chemotherapeutic resistance by targeting EIF4A1, reducing JUN and MMP12, and increasing MYC. Previous studies have shown that EIF4A1 is inhibited by PDCD4, while PDCD4 regulates the MYC and JUN pathways $(32,33)$. This identified an uncommon integrated network, the miR-1284/EIF4A1/JUN/MYC signaling pathway. Reducing the permeability glycoprotein (P-gp), a multidrug resistance protein that is created by MDR1, and downregulating MDR1 play an indispensable role in circumventing MDR in cancer. Studies have shown that inhibiting JUN significantly reduces the degree of MDR by decreasing MDR1 and P-gp (34), leading to inefficient transport that keeps a lower concentration of drugs inside the cancer cells, which reverses MDR. As previously described, another primary reason for MDR is to reduce susceptibility to drug-induced apoptosis $(21,22)$. Fu et al (35) confirmed that a reduced expression of JUN contributes to an extended G1 phase and induces apoptosis. In addition, activating MYC has been proved to generate apoptosis by triggering cytochrome $c$ before activating caspase (36).

JUN, a binding complex that is correlated with the MMP12 booster, directly activities MMP12 and belongs to the matrix metalloproteinases (MMPs) that have been known to act in an indispensable manner in tumor invasiveness and metastasis $(37,38)$. MMP12 has been reported to be connected to tumor metastasis, such as lung carcinoma and squamous carcinomas of the head and neck $(39,40)$. A recent study also showed that silencing MMP12 suppresses the proliferation and invasion of lung cancer cells (41). Interestingly, JUN increases tumor migration and invasion activity (42), and GC cell metastasis is suppressed by reducing JUN and MMP12. Primary tumors and tumors with metastasis are often different from MDR cells with metastasis because of the stronger 
drug-resistance (43). Inhibiting metastasis may be another key mechanism by which MDR in tumor cells is circumvented.

In this study, miR-1284 was regulated to reverse MDR in GC cells by downregulating EIF4A1 in vitro and in vivo. This study is the first to demonstrate a connection between miR-1284 and EIF4A1, an important member of the EIF family, and demonstrates the power of miR-1284 in regulating MDR development. Moreover, this study draws our attention to EIF4A1 as an underlying therapeutic target for GC and proposes new concepts whereby miR-1284 can suppress cancer progression.

\section{Acknowledgements}

The present study was supported by grants from the National Natural Science Foundation of China (no. 30860273; no. 81060201); the Key Health Science Foundation of Guangxi (no. 14124004-1-9), the Natural Science Foundation of Guangxi (no. 2015GXNSFDA227001) and Innovation Project of Guangxi Graduate Education.

\section{References}

1. Baba H, Kuwabara K, Ishiguro T, Kumamoto K, Kumagai Y, Ishibashi K, Haga N and Ishida H: Prognostic factors for stage IV gastric cancer. Int Surg 98: 181-187, 2013.

2. Fan D, Zhang X, Chen X, Mou Z, Hu J, Zhou S, Ding J and Wu K: Bird's-eye view on gastric cancer research of the past 25 years. J Gastroenterol Hepatol 20: 360-365, 2005.

3. Jemal A, Siegel R, Xu J and Ward E: Cancer statistics, 2010. CA Cancer J Clin 60: 277-300, 2010.

4. Fodale V, Pierobon M, Liotta L and Petricoin E: Mechanism of cell adaptation: When and how do cancer cells develop chemoresistance? Cancer J 17: 89-95, 2011.

5. Dai Z, Huang Y and Sadée W: Growth factor signaling and resistance to cancer chemotherapy. Curr Top Med Chem 4: 1347-1356, 2004.

6. Bartel DP: MicroRNAs: Genomics, biogenesis, mechanism, and function. Cell 116: 281-297, 2004.

7. Calin GA, Sevignani C, Dumitru CD, Hyslop T, Noch E, Yendamuri S, Shimizu M, Rattan S, Bullrich F, Negrini M, et al Human microRNA genes are frequently located at fragile sites and genomic regions involved in cancers. Proc Natl Acad Sci USA 101: 2999-3004, 2004.

8. Zheng T, Wang J, Chen X and Liu L: Role of microRNA in anticancer drug resistance. Int J Cancer 126: 2-10, 2010.

9. Hummel R, Hussey DJ and Haier J: MicroRNAs: Predictors and modifiers of chemo- and radiotherapy in different tumour types. Eur J Cancer 46: 298-311, 2010.

10. Ma J, Dong C and Ji C: MicroRNA and drug resistance. Cancer Gene Ther 17: 523-531, 2010.

11. An Y, Zhang Z, Shang Y, Jiang X, Dong J, Yu P, Nie Y and Zhao Q: miR-23b-3p regulates the chemoresistance of gastric cancer cells by targeting ATG12 and HMGB2. Cell Death Dis 6: e1766, 2015.

12. Zhu W, Shan X, Wang T, Shu Y and Liu P: miR-181b modulates multidrug resistance by targeting BCL2 in human cancer cell lines. Int J Cancer 127: 2520-2529, 2010.

13. Zhu W, Zhu D, Lu S, Wang T, Wang J, Jiang B, Shu Y and Liu P: miR-497 modulates multidrug resistance of human cancer cell lines by targeting BCL2. Med Oncol 29: 384-391, 2012.

14. Patnaik SK, Yendamuri S, Kannisto E, Kucharczuk JC, Singhal S and Vachani A: MicroRNA expression profiles of whole blood in lung adenocarcinoma. PLoS One 7: e46045, 2012.

15. Chen W, Tang Z, Sun Y, Zhang Y, Wang X, Shen Z, Liu F and Qin X: miRNA expression profile in primary gastric cancers and paired lymph node metastases indicates that miR-10a plays a role in metastasis from primary gastric cancer to lymph nodes. Exp Ther Med 3: 351-356, 2012.

16. Yasui W, Sentani K, Sakamoto N, Anami K, Naito Y and Oue N: Molecular pathology of gastric cancer: Research and practice. Pathol Res Pract 207: 608-612, 2011.
17. Lu D, Xiao Z, Wang W, Xu Y, Gao S, Deng L, He W, Yang Y, Guo $X$ and Wang X: Down regulation of CIAPIN1 reverses multidrug resistance in human breast cancer cells by inhibiting MDR1. Molecules 17: 7595-7611, 2012.

18. Rebucci M and Michiels C: Molecular aspects of cancer cell resistance to chemotherapy. Biochem Pharmacol 85: 1219-1226, 2013.

19. Baguley BC: Multiple drug resistance mechanisms in cancer. Mol Biotechnol 46: 308-316, 2010.

20. Butler EB, Zhao Y, Muñoz-Pinedo C, Lu J and Tan M: Stalling the engine of resistance: Targeting cancer metabolism to overcome therapeutic resistance. Cancer Res 73: 2709-2717, 2013.

21. Johnstone RW, Ruefli AA and Lowe SW: Apoptosis: A link between cancer genetics and chemotherapy. Cell 108: 153-164, 2002.

22. Rabik CA and Dolan ME: Molecular mechanisms of resistance and toxicity associated with platinating agents. Cancer Treat Rev 33: 9-23, 2007.

23. Wu Q, Yang Z, Xia L, Nie Y, Wu K, Shi Y and Fan D: Methylation of miR-129-5p CpG island modulates multi-drug resistance in gastric cancer by targeting $\mathrm{ABC}$ transporters. Oncotarget 5: 11552-11563, 2014.

24. Shang Y, Zhang Z, Liu Z, Feng B, Ren G, Li K, Zhou L, Sun Y, Li M,Zhou J, et al: miR-508-5p regulates multidrug resistance of gastric cancer by targeting ABCB1 and ZNRD1. Oncogene 33: 3267-3276, 2014

25. Wang Y, Gu X, Li Z, Xiang J, Jiang J and Chen Z: microRNA expression profiling in multidrug resistance of the 5-FU-induced SGC-7901 human gastric cancer cell line. Mol Med Rep 7: 1506-1510, 2013.

26. Bordeleau ME, Robert F, Gerard B, Lindqvist L, Chen SM, Wendel HG, Brem B, Greger H, Lowe SW, Porco JA Jr, et al: Therapeutic suppression of translation initiation modulates chemosensitivity in a mouse lymphoma model. J Clin Invest 118: 2651-2660, 2008.

27. Hagner PR, Schneider A and Gartenhaus RB: Targeting the translational machinery as a novel treatment strategy for hematologic malignancies. Blood 115: 2127-2135, 2010.

28. Svitkin YV, Pause A, Haghighat A, Pyronnet S, Witherell G, Belsham GJ and Sonenberg N: The requirement for eukaryotic initiation factor 4A (elF4A) in translation is in direct proportion to the degree of mRNA 5' secondary structure. RNA 7: 382-394, 2001.

29. Shuda M, Kondoh N, Tanaka K, Ryo A, Wakatsuki T, Hada A, Goseki N, Igari T, Hatsuse K, Aihara T, et al: Enhanced expression of translation factor mRNAs in hepatocellular carcinoma. Anticancer Res 20: 2489-2494, 2000.

30. Eberle J, Krasagakis K and Orfanos CE: Translation initiation factor eIF-4A1 mRNA is consistently overexpressed in human melanoma cells in vitro. Int J Cancer 71: 396-401, 1997.

31. Modelska A, Turro E, Russell R, Beaton J, Sbarrato T, Spriggs K, Miller J, Gräf S, Provenzano E, Blows F, et al: The malignant phenotype in breast cancer is driven by eIF4A1-mediated changes in the translational landscape. Cell Death Dis 6: e1603, 2015.

32. Lankat-Buttgereit B and Göke R: The tumour suppressor Pdcd4: Recent advances in the elucidation of function and regulation. Biol Cell 101: 309-317, 2009.

33. Zhen Y, Liu Z, Yang H, Yu X, Wu Q, Hua S, Long X, Jiang Q, Song Y, Cheng C, et al: Tumor suppressor PDCD4 modulates miR-184-mediated direct suppression of C-MYC and BCL2 blocking cell growth and survival in nasopharyngeal carcinoma. Cell Death Dis 4: e872, 2013.

34. Sui H, Zhou S, Wang Y, Liu X, Zhou L, Yin P, Fan Z and Li Q: COX-2 contributes to P-glycoprotein-mediated multidrug resistance via phosphorylation of c-Jun at Ser63/73 in colorectal cancer. Carcinogenesis 32: 667-675, 2011.

35. Fu Y, Lin Y, Yang Z, Yang G, Li G, Liu Y, Tan X, Huang Y, Wu X, Wang Y, et al: FBXW7 overexpression suppresses renal cancer cell proliferation and induces apoptosis. Med Oncol 32: 215, 2015.

36. Juin P, Hueber AO, Littlewood T and Evan G: c-Myc-induced sensitization to apoptosis is mediated through cytochrome c release. Genes Dev 13: 1367-1381, 1999.

37. Wu L, Tanimoto A, Murata Y, Sasaguri T, Fan J, Sasaguri Y and Watanabe T: Matrix metalloproteinase-12 gene expression in human vascular smooth muscle cells. Genes Cells 8: 225-234, 2003.

38. Xie S, Issa R, Sukkar MB, Oltmanns U, Bhavsar PK, Papi A, Caramori G, Adcock I and Chung KF: Induction and regulation of matrix metalloproteinase-12 in human airway smooth muscle cells. Respir Res 6: 148, 2005. 
39. Hofmann HS, Hansen G, Richter G, Taege C, Simm A, Silber RE and Burdach S: Matrix metalloproteinase-12 expression correlates with local recurrence and metastatic disease in non-small cell lung cancer patients. Clin Cancer Res 11: 10861092, 2005.

40. Kim JM, Kim HJ, Koo BS, Rha KS and Yoon YH: Expression of matrix metalloproteinase-12 is correlated with extracapsular spread of tumor from nodes with metastasis in head and neck squamous cell carcinoma. Eur Arch Otorhinolaryngol 270: $1137-1142,2013$

41. Lv FZ, Wang JL, Wu Y, Chen HF and Shen XY: Knockdown of MMP12 inhibits the growth and invasion of lung adenocarcinoma cells. Int J Immunopathol Pharmacol 28: 77-84, 2015 .
42. Toft DJ, Rosenberg SB, Bergers G, Volpert O and Linzer DI: Reactivation of proliferin gene expression is associated with increased angiogenesis in a cell culture model of fibrosarcoma tumor progression. Proc Natl Acad Sci USA 98: 13055-13059, 2001.

43. O'Shaughnessy J: Extending survival with chemotherapy in metastatic breast cancer. Oncologist 10 (Suppl 3): 20-29, 2005. 